sem Zahlenwert legen wir allerdings im Hinblick auf die Meßgenauigkeit unserer derzeitigen Apparatur kein zu großes Gewicht bei. Zudem ist zu erwarten, $\mathrm{da} ß$ er für jeden einzelnen Vervielfacher anders ist.

Nimmt man für die Photokathode einen Wirkungs. grad von 1 Primärelektron auf 100 Lichtquanten an, so folgt, daß jedes $\alpha$-Teilchen in Ar der vorliegenden Reinheit etwa 1000 beobachtbare Lichtquanten mit einer Wellenlänge zwischen 3000 und $4000 \AA$ erzeugt. Die auf diese Weise ausgestrahlte Energie ist etwa der 1000-ste Teil derjenigen Energie, die von dem $\boldsymbol{a}$-Teilchen in Form von Anregungsenergie an das Gas abgegeben wird. Für diese geringe Ausbeute sehen wir eine Erklärung darin:

Bei Normaldruck ist die Wechselwirkung angeregter Teilchen (Atome oder Moleküle) mit gleichartigen Teilchen im Grundzustand stark und begünstigt strahlungslose Vernichtung der Anregungsenergie, sogenannte „Konzentrationslöschung“.

Die Beobachtung von „Resonanzquanten“, die bei der Rückkehr der Teilchen in ihren Elektronengrund- zustand emittiert werden, ist in reinen Gasen von höherem Druck nicht möglich, und zwar, abgesehen von dem meßtechnischen Grund, daß sie bei den meisten Gasen weit im UV liegen, prinzipiell nicht. Es sind dieselben Ursachen, die auch die Selbstumkehr der Resonanzlinien und die Diffusion der Resonanzstrahlung bewirken.

Wegen der starken Wechselwirkung angeregter Teilchen mit gleichartigen Nachbarn im Grundzustand ist es nicht mehr sinnvoll, den Anregungszustand auf ein einzelnes Teilchen zu lokalisieren; es ist vielmehr so, daß sich die Wahrscheinlichkeit für die Emission des Lichtquants auf eine größere Zahl von Molekülen verteilt, wenn nicht isolierte metastabile Zustände beteiligt sind. Im ersteren Falle besteht Aussicht auf eine Steigerung der Lichtausbeute durch Sensibilisatoren, in ähnlicher Weise wie dies bei einigen organischen Flüssigkeiten ${ }^{6}$ beobachtet wurde. Diese Erscheinungen sollen $u$. a. weiter untersucht werden.

6 H. K a $11 \mathrm{~m}$ a n n, M. Furst, Physic. Rev. 79, 857 [1950].

\title{
Messung von Gasdichten mit Korpuskularstrahlsonden
}

\author{
Von E. SCHOPPER und B: SCHUMACHER \\ Aus dem Physikalischen Institut der Technischen Hochschule Stuttgart \\ (Z. Naturforschg. 6 a, 700-705 [1951]; eingegangen am 9. August 1951) \\ Erich Regener zum 70. Geburtstag
}

\begin{abstract}
Für die Messung nichtstationärer Zustände der Dichte stark verdünnter Gase, bei denen Schlieren- und Interferenzverfahren versagen, läßt sich die Absorption eines Korpuskularstrahlbündels verwenden. Es werden die allgemeinen Gesichtspunkte für die Anwendung korpuskularer Strahlsonden diskutiert: Bei Ionensonden ist die Schwächung vorwiegend durch den Energieverlust der Einzelpartikel gegeben. Die für Protonen für die erwähnten Meßprobleme benötigten Strahlspannungen liegen bei $100-200 \mathrm{kV}$. Das geometrische Auflösungsvermögen von Protonensonden ist wegen der relativen Kleinheit der Streueffekte praktisch gleich dem Bündelquerschnitt. Bei Elektronensonden ist die Schwächung überwiegend durch Absorption und Streuung verursacht und äußert sich in einer Änderung der Teilchenzahl. Der Schwächungskoeffizient läßt sich dem Meßproblem durch die Wahl der Strahlspannung bequem anpassen. Bei inhomogener Dichteverteilung im Meßfeld ist die aus der Strahlschwächung ermittelte integrale Dichte nur dann eindeutig, wenn der durch die Streueffekte verbreiterte Strahlquerschnitt, der „Trübungsquerschnitt“, an der Messung beteiligt wird. Dadurch ist auch das geometrische Auflösungsvermögen der Elektronensonden gegeben. Es wird über die praktische Ausführung einer Apparatur berichtet, die mit Hilfe einer durch Düsen gebildeten Zwischenkammer ohne Folienfenster enge monoenergetische Korpuskularbündel, z. B. Elektronen $\geqq 3 \mathrm{keV}$, liefert und einen Außendruck bis 1,5 atm zuläßt. Es werden zwei Meßbeispiele mit Elektronensonden wiedergegeben: der Dichteverlauf beim Austritt eines schnellen Luftstrahles in ein Unterdruckgebiet und der Dichteverlauf in einer Gebläseflamme unter Atmosphärendruck.
\end{abstract}

$\mathrm{D}^{\mathrm{s}}$ ie Messung nicht stationärer Zustände von Temperatur und Druck stark verdünnter Gase, ein Problem, das z. B. bei der Erforschung der Luftschichten der hohen Atmosphäre auftritt, stößt auf fol- gende Schwierigkeiten: Bei Temperaturmessungen mit Thermometerorganen, die einen Wärmeaustausch mit dem Gas voraussetzen, tritt das Problem der Ventilation auf. In schnell bewegten Gasen sind aerodyna- 
mische Effekte wirksam, die bei Temperatur- und Druckmessungen berücksichtigt werden müssen.

Im Rahmen eines Programms zur Untersuchung der Luftschichten der hohen Atmosphäre unter der Leitung von E. Regener ${ }^{1}$, das auch die Entwicklung und Untersuchung von Instrumenten zur Druck- und Temperaturmessung ${ }^{2,3}$ unter diesen Verhältnissen erforderte, zeigte sich deutlich, daß die Brauchbarkeit solcher Messungen wesentlich von der Zuverlässigkeit abhängt, mit der die aerodynamischen Korrekturen erfaßt werden können. In Gasen von geringem Druck sind aber die Verhältnisse bislang wenig untersucht. Insofern sind z. B. auch bei amerikanischen Messungen $^{4}$ mit Raketen vom Jahre 1947 noch relativ große Fehlergrenzen angegeben.

Die üblichen aerodynamischen Meßverfahren zur Untersuchung von Strömungsfeldern von Gasen mit Interferometer- und Schlierenmethoden` versagen im Bereich geringer Drucke. Es erschien aussichtsreich, die Dichteverteilung in einem solchen Strömungsfeld mit Hilfe einer Korpuskularstrahlsonde zu bestimmen, wobei die Absorption des Korpuskularbündels in der Meßstrecke ein Maß für die Dichte ergibt. Darüber hinaus lassen sich die bisherigen Thermometerorgane überhaupt durch die Korpuskularsonde ersetzen. In Verbindung mit einer Druckmessung liefert die Dichtemessung die gesuchten Zustandsgrößen, und zwar bei geeigneter Ausführung der Meßstrecke ohne Einfluß der aerodynamischen Effekte und der Ventilationsgröße. Auch bei Messungen in Gasen hoher Temperatur im Plasmazustand kann die Korpuskularsonde wertvoll sein.

Es wurde bisher eine $\alpha$-Strahlsonde ${ }^{6,8}$ für Temperaturmessungen in der Atmosphäre und eine stationäre Apparatur ${ }^{7,8}$ zur Vermessung von Strömungsfeldern mit Elektronen- bzw. Protonenbündeln aufgebaut. Wir berichten im folgenden über die prinzipiellen Möglichkeiten der Anwendung von Strahlsonden und über Meßbeispiele mit der letztgenannten Apparatur.

\section{Allgemeine Gesichtspunkte für Strahlsonden}

Wir betrachten ein Strahlbündel von geringem Querschnitt, das wir als Strahlsonde bezeichnen und das aus Korpuskeln (Ionen, Elektronen) bestehe.

1 E. Regener, H.-K. Pa etzold, W. Rau u. E. S c h o p p e r, Manuskript 1944.

2 E. R e g e n e r, Veröff. Deutsche Luftfahrtakademie 1942.

3 E. Schopper, Temperaturmessung in schneller Strömung, Manuskript 1944.

4 N. B est, R. Havens u. H. LaGow, Physic. Rev. (2) 71, 915 [1947].
In einem korpuskularen Strahl mit der Energie $\varepsilon$ der einzelnen Strahlpartikel und der Zahl $n$ der Partikeln je Flächeneinheit und Sekunde ist der Energiefluß je Flächeneinheit

$$
E=\varepsilon n
$$

und die Intensität des Gesamtstrahls mit der Querschnittsfläche $f$

$$
J=n f .
$$

Die Schwächung des Energieflusses je Masseneinheit längs des Weges $s$ rührt von zwei Anteilen her:

$$
\frac{\mathrm{d} E}{\mathrm{~d}(\varrho s)}=\frac{n \partial \varepsilon}{\partial(\varrho s)}+\begin{gathered}
\varepsilon \partial n \\
\partial(\varrho s)
\end{gathered},
$$

wenn $\varrho$ die Gasdichte ist.

Je nach dem Schwächungsmechanismus überwiegt der eine oder der andere der beiden Terme.

a) I o n e n s o n d e n

Für schwere Teilchen, $\alpha$-Teilchen und Ionenstrahlen ist

$$
n \frac{\partial \varepsilon}{\partial(\varrho s)} \gg \frac{\varepsilon \partial n}{\partial(\varrho s)} .
$$

Das Meßverfahren läuft auf eine Bestimmung der Teilchenenergie hinter der Meßstrecke hinaus. Der Energieverlust entspricht einer Reichweiteänderung, die meßtechnisch in bekannter Weise durch die Ionisation (oder Lumineszenzeffekte) längs der nach Durchlaufen der Meßstrecke vorhandenen Restreichweite des Teilchens erfaßt wird. Es ist wesentlich, daß in der Meßstrecke gebildete Ionen die Messung nicht beeinflussen können, da diese je nach der Strömungsgeschwindigkeit des Gases mehr oder weniger weggeführt werden. Hierin liegt der Unterschied zu dem als Alphatron ${ }^{9}$ bekannten Manometer, bei dem die differentielle Ionisierung am Anfang der Bahn als Funktion des Druckes gemessen wird. In strömendem Gas wird die Anzeige des Alphatrons falsch.

Wie aus Tab. 1 hervorgeht ${ }^{10}$, erfordert eine Protonensonde für Messungen bei einem Druck von etwa

5 W. W a e h l, Zusammenfass. Darstellung in Arch. techn. Mess. 180 [1951], Z. 721.

6 D. K n a p p, Diplom-Arbeit, Stuttgart 1950.

7 B. S c h u m a c h er, Diplom-Arbeit, Stuttgart 1950.

8 E.S chopper, B. S chum a cher u. D. Knapp, Vortrag Physikertagung Bad Nauheim, Oktober 1950 und Physik. Ber. 30, 420 [1951].

9 J. R. D ow n ing u. G. L. M elle n, Rev. sci. Instruments 17, 218 [1946]; Ind. Engng. Chem. 40, 787 [1948]; R. S h e p a rd, Rev. sci. Instruments 10, 181 [1939].

10 Living st on - B e the, Rev. mod. Physics 9, 268 [1937]. 


\begin{tabular}{|c|c|c|c|c|}
\hline \multirow{2}{*}{$\underset{\mathrm{keV}}{\text { Energie }}$} & \multicolumn{4}{|c|}{$\begin{array}{l}\text { Reichweite in cm in Luft von } 273^{\circ} \mathrm{K} \text { für } \\
\text { Protonen } \\
\alpha \text {-Teilchen }\end{array}$} \\
\hline & $\begin{array}{c}\text { bei } 760 \\
\text { Torr }\end{array}$ & $\begin{array}{c}\text { bei } 10 \\
\text { Torr }\end{array}$ & $\begin{array}{c}\text { bei } 760 \\
\text { Torr }\end{array}$ & $\begin{array}{l}\text { bei } 10 \\
\text { Torr }\end{array}$ \\
\hline 100 & 0,12 & 9 & 0,1 & 7,6 \\
\hline 200 & 0,30 & 23 & 0,17 & 13 \\
\hline 500 & 0,85 & 64 & 0,34 & 26 \\
\hline
\end{tabular}

Tab. 1. Reichweiten von $\alpha$-Teilchen und Protonen verschiedener Energien.

10 Torr, einer Temperatur von $273^{\circ} \mathrm{K}$ und einer Meßstrecke von der Länge $s=5 \mathrm{~cm}$ eine Protonenenergie von 100 bis $200 \mathrm{kV}$; diese können ohne großen Aufwand mit einem Kanalstrahlrohr erzeugt werden.

Die Relativempfindlichkeit der Sonde für Dichteänderungen wird naturgemäß um so größer, je größei $\frac{\mathrm{d} \varepsilon}{\mathrm{d}(o s)}$ ist. Über diese durch die Braggsche Kurve und durch Rechnungen von $\mathrm{B}$ e th $\mathrm{e}^{\mathbf{1 0}}$ und $\mathrm{B} \mathrm{l}$ o $\mathrm{ch}$ quantitativ bekannte Größe läßt sich qualitativ folgendes sagen: Im Bereich großer Werte, die eine große Empfindlichkeit ergeben, ist auch ihre Änderung mit dem Weg $s$ groß. Bei homogener Dichte in der Meßstrecke ist trotzdem der integral gemessene Energieverlust ein eindeutiges $\mathrm{Ma} ß$ der Dichte.

Ein solcher Fall liegt vor bei unserer als Thermometer entwickelten $\alpha$-Sonde ${ }^{6,8}$ : Sie erlaubte bei Normaldruck Dichteänderungen von $1,5 \%$ bzw. Temperaturänderungen von $0,5^{\circ} \mathrm{C}$ sicher zu messen; die Empfindlichkeit und die Zeitkonstante der Anzeige von der Größenordnung $10^{-2} \mathrm{sec}$ waren hierbei durch die statistischen Schwankungen der $\alpha$-Strahlung begrenzt. Bei inhomogener Dichte längs der Meßstrecke z. B. einer „Dichteschliere“ würde bei veränderlichem $\mathrm{d} \varepsilon / \mathrm{d} s$ die Messung geometrie-abhängig. In diesem Falle muß in solchen Bereichen der Teilchenenergie $\varepsilon$ gemessen werden, in denen $\mathrm{d} \varepsilon / \mathrm{d} s$ sich wenig ändert; dann ist allerdings auch $\mathrm{d} \varepsilon / \mathrm{d} s$ klein und die Dichteempfindlichkeit des Meßverfahrens geringer. Das geometrische Auflösungsvermögen von Ionensonden ist infolge der Kleinheit der Streueffekte praktisch gleich dem Bündelquerschnitt, der sehr klein gemacht werden kann.

b) Elektronensonden

Der Schwächungsmechanismus in einem Elektronenbündel in dem für unser Problem in Frage kommenden Bereich der Teilchenenergie ist dadurch charakterisiert, daß der Energieverlust der Einzelpartikel nur unwesentlich an der Schwächung beteiligt ist gegenüber der Änderung der Teilchenzahl; es ist

$$
\frac{n \partial \varepsilon}{\partial(\varrho s)} \ll \frac{\varepsilon \partial n}{\partial(\varrho s)} .
$$

Das Meßverfahren läuft auf eine Bestimmung der Teilchenzahl, z. B. durch Messung des Ladungstransports, hinaus. An der Änderung der Teilchenzahl ist

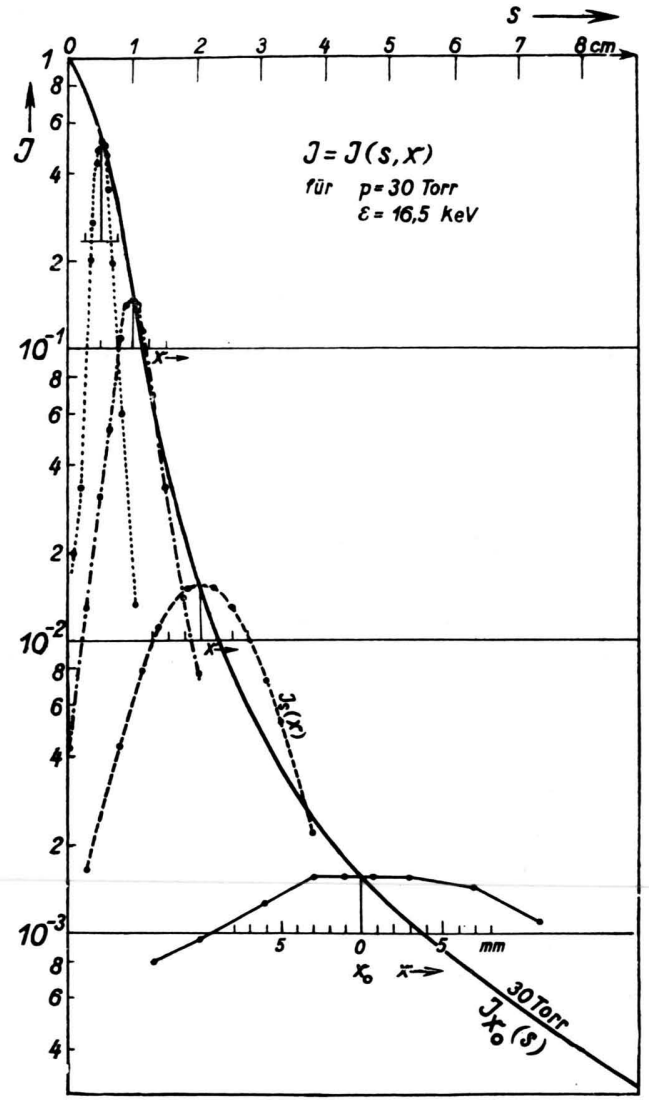

Abb. 1. Intensität eines Elektronenstrahls in Luft längs und quer zur Strahlachse.

auf jeden Fall echte Absorption, d. h. die katastrophische Abbremsung eines Teilchens, beteiligt; außerdem wirken, abhängig von der Geometrie der Meßanordnung, die Streueffekte mit. Diese bewirken bekanntlich, daß ein enges Parallelbündel von Elektronen sich beim Durchgang durch Materie längs seines Weges $s$ diffus verbreitert. Die Verhältnisse erläutert Abb. 1: Wir messen mit einem Auffänger vom Durchmesser $d_{\mathrm{A}}=1,5 \mathrm{~mm}$ die Intensität $J(s)$ des Strahles in Abhängigkeit vom Weg $s$, und die Intensitätsverteilung $J_{s}(x)$ bei festem $s$ in Richtung $x$ quer zur Strahlrichtung. Nach einem Weg von $2 \mathrm{~cm}(p=30$ Torr $)$ 


\begin{tabular}{|c|c|c|c|c|}
\hline $\begin{array}{l}\text { Energie bzw. } \\
\text { Wellenlänge }\end{array}$ & $\begin{array}{l}a_{0}=a_{0} / Q \\
\text { in } \mathrm{cm}^{2} / \mathrm{g}\end{array}$ & $\begin{array}{l}\alpha_{\varrho} \text { in } \mathrm{cm}^{-1} \mathrm{f} \\
\text { bei } 100 \text { Torr }\end{array}$ & $\begin{array}{l}\text { von } 273^{\circ} \mathrm{K} \\
\text { bei } 10 \mathrm{Torr}\end{array}$ & Anmerkungen \\
\hline $\begin{array}{c}\text { Elektronen } \\
1,6 \mathrm{keV} \\
5,8 \\
10,5 \\
16,7 \\
24,7\end{array}$ & $\begin{array}{l}1,4 \cdot 10^{6} \\
1,5 \cdot 10^{5} \\
3,6 \cdot 10^{4} \\
8,6 \cdot 10^{3} \\
2,9 \cdot 10^{3}\end{array}$ & $\begin{array}{l}240 \\
25 \\
6,2 \\
1,43 \\
0,5\end{array}$ & $\begin{array}{c}24 \\
2,5 \\
0,62 \\
0,14 \\
0,05\end{array}$ & \\
\hline $\begin{array}{l}\text { Licht } \\
\qquad \begin{array}{c}7,4 \mu \\
5,7 \\
9,5 \\
2537 \AA \\
1\end{array}\end{array}$ & $\begin{array}{l}\text { ca. } 600 \text { für } \mathrm{SO}_{2} \\
\text { ca. } 300 \text { für } \mathrm{NO}_{2} \\
\text { ca. } 40 \text { für } \mathrm{O}_{3} \\
\text { ca. } 1,5 \cdot 10^{5} \text { für } \mathrm{O}_{3} \\
\text { ca. } 3,1 \cdot 10^{5} \text { für } \mathrm{O}_{2}\end{array}$ & $\begin{array}{l}\text { für das } \\
- \\
- \\
- \\
-\end{array}$ & $\begin{array}{l}\text { bene Gas } \\
0,023 \\
0,01 \\
0,001 \\
4,2 \\
2 \cdot 10^{-5}\end{array}$ & $\begin{array}{l}\text { keine lineare } \\
\text { Abhängigkeit } \\
\text { von der } \\
\text { Dichte }\end{array}$ \\
\hline
\end{tabular}

Tab. 2. Vergleich der Absorptionskoeffizienten verschiedener Strahlenarten 12,13.

ist der Strahl, der ursprünglich einen Durchmesser von $0,2 \mathrm{~mm}$ hatte, schon merklich verbreitert: in $x=10 \mathrm{~mm}$ Abstand von der Achse beträgt seine Intensität $J_{\mathrm{s}}(x)$ noch $10 \%$ der Intensität $J_{\mathrm{s}}\left(x_{0}\right)$ in der Achse. Wir haben Teilchenverlust durch Streuung. Der empirische Schwächungskoeffizient ist also stark geometrie-abhängig.

Hinter einer gewissen Materieschicht $\varrho s_{\vartheta}\left(s_{\vartheta} \approx 4 \mathrm{~cm}\right.$ unter den Bedingungen von Abb.3) wird die $J(s)$ Kurve wesentlich flacher (Diffusitätsgebiet). Infolge des Zusammenwirkens von Absorption und Streuung wird nämlich die Intensitätsverteilung $J(x)$ über einen gewissen Bereich $x_{c}$ von $x$ konstant (z. B. ist $x_{c} \approx 5 \mathrm{~mm}$ für $\mathrm{s}=4 \mathrm{~cm}$ in Abb. 3). In einer Kernzone dieses Bereiches herrscht Gleichgewicht zwischen den einwärts- und auswärtsgestreuten Elektronen. Damit wird der Streuanteil an der Schwächung des Energieflusses in dieser Kernzone geometrie-unabhängig. Die Schwächung der Intensität, gemessen in dieser Kernzone, kann im Bereich $s>s_{\vartheta}$ formal in guter Näherung durch ein Exponentialgesetz dargestellt werden. Für ein Elektronenbündel, dessen Intensität bei $s=s^{\vartheta}$ den Betrag $J_{s_{\vartheta}}$ hat, ist die Intensität $J(\varrho, s)$ hinter einer homogenen Materieschicht der Dichte $\varrho$ und der Dicke $s>s_{\vartheta}$

$$
J(\varrho, s)=J_{s_{\vartheta}} \cdot \exp \left\{-\alpha_{Q}\left(s-s_{\vartheta}\right)\right\} .
$$

Der Schwächungskoeffizient $\alpha_{\varrho}$, der sogenannnte praktische Absorptionskoeffizient, ist eine Funktion der Teilchenenergie $\varepsilon$, hängt in geringem Maße vom

11 W i e n- H a r m s, Hdb. Exp. Physik, Bd. 14 und 15.

12 K o h l ra s c h, Prakt. Physik, 19. Aufl., Tab. 48.

13 Landolt-Börnstein, Physik.-chem. Tab. I, 1. Berlin-Göttingen-Heidelberg 1950.
Atomgewicht des absorbierenden Gases $\mathrm{ab}^{11}$ und ist der Dichte $\varrho$ direkt proportional, so daß er in der Form $\alpha_{\varrho}(\varepsilon)=\alpha_{0}(\varepsilon) \varrho$ geschrieben werden kann.

Wir betrachten jetzt eine fest vorgegebene Meßstrecke $s=d$. Ändert sich die Dichte im Bereich $s_{\vartheta} \leq s \leq d$ um den mittleren Betrag $\overline{\Delta \varrho}$, bezogen auf die Strecke $d-s_{\vartheta}$, so gilt für die Änderung der bei $d$ gemessenen Intensität

$$
\ln (1+\Delta J / J)=-\alpha_{\varrho}(1+\overline{\Delta \varrho} / \varrho)\left(d-s_{\vartheta}\right)
$$

bzw. für kleine Änderungen von $\varrho$ :

$$
\Delta J / J=-c_{\varrho} \overline{\Delta Q} / \varrho\left(d-s_{\vartheta}\right) .
$$

Da $\alpha_{o}(\varepsilon)$ durch die Strahlspannung in weiten Grenzen wählbar ist, kann für jede Dichte $\varrho$ eine große Relativempfindlichkeit erzielt werden (Tab. 2).

Für die Dichteänderungen im Strahlbereich $s<s_{\text {i }}$ sind die Streueffekte geometrie-abhängig. Dies gilt für sehr große Dichtegradienten (z. B. durch Folien verursacht) und bei stark verschiedenem Atomgewicht der Dichteschliere auch im Strahlbereich $s>s_{\vartheta}$.

Die Streueffekte begrenzen auch das geometrische Auflösungsvermögen der Elektronensonden quer zur Strahlrichtung. Die gemessene Dichteänderung ist ein Mittelwert über den Trübungsbereich; wir bezeichnen so das Gebiet, aus dem gestreute Elektronen den Auffänger erreichen. Infolge der auf die Streuelektronen wirksamen Absorption ist dieses Einzugsgebiet senkrecht zur Strahlrichtung von der Größenordnung de: sogenannten Rückdiffusionsdicke $\delta^{11}$. Diese wird mit steigender Absorption $\alpha_{0}$ kleiner. Messungen und theoretische Abschätzungen der Rückdiffusionsdicke liegen nur für schnelle Elektronen in Absorbern hohen 
Atomgewichtes vor ${ }^{11}$. Extrapoliert man die Werte für Verhältnisse, unter denen unsere Messungen in Abb. 3 gemacht wurden, so ergibt sich ein Wert $\delta \simeq 2 \mathrm{~mm}$, in Übereinstimmung mit den experimentellen Beobachtungen (Kurve für $p=p_{0}$ in Abb. 3). Eingehende Untersuchungen zu diesem Fragenkomplex und die Behandlung auf Grund neuerer Rechnungen ${ }^{14,15}$ sind im Gange.

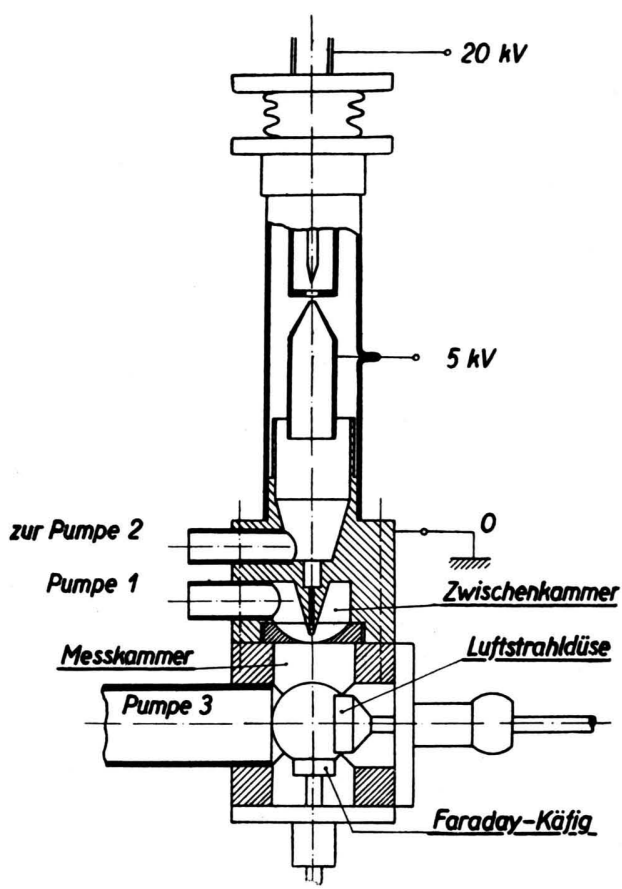

Abb. 2. Schematischer Aufbau der Elektronensonde.

\section{Experimentelle Ergebnisse mit Elektronensonden}

Der Sondenstrahl wurde mit einer schematisch in Abb. 2 dargestellten Anordnung erzeugt. Der Strahl tritt, aus dem Hochvakuum kommend, über eine Zwischenkammer durch zwei Düsen in die Meßkammer ein. Das aus der Meßkammer in entgegengesetzter Richtung strömende Gas wird einmal in der Zwischenkammer zwischen den Düsen, der Rest aus dem Hochvakuumrohr selbst abgesaugt. Die zylindrischen Düsen hatten 0,3 mm Durchmesser auf $2 \mathrm{~mm}$ Länge bzw. 0,05 mm Durchmesser auf 0,1 mm.

Größere Düsendurchmesser erlaubten die seinerzeit verwendeten Quecksilberpumpen nicht. Mit der in Abb. 2 schematisch gezeigten Spitz-Anode gelang es, durch diese engen Düsen einen Sondenstrahl mit ausreichender Intensität $\mathrm{zu}$ bekommen. Er hatte im Hochvakuum eine Intensität von $120 \mu \mathrm{A}$ bei $20 \mathrm{kV}$ Strahlspannung und eine Stromdichte von $60 \mu \mathrm{A} / \mathrm{mm}^{2}$ im Zentrum; hinter

14 G. M o li è r e, Z. Naturforschg. 2a, 133 [1947].

15 E. J. Williams, Physic. Rev. 58, 292 [1940]. der Austrittsdüse in die Meßkammer war die Intensität $0,1 \mu \mathrm{A}$. Der Druck in der Meßkammer konnte von 0-70 Torr variiert werden, ohne Rückwirkung auf das Hochvakuumrohr. Noch bei $3 \mathrm{kV}$ Beschleunigungsspannung am Rohr war die Strahlintensität für Messungen ausreichend, so daß auch weiche monochromatische Sonden zur Verfügung standen, wie sie durch ein Lenard-Fenster nicht zu erhalten, aber für Messungen bei Drucken von 1 Torr erforderlich sind. Inzwischen hat der eine von uns (B.Sch.) eine verbesserte Apparatur aufgestellt, die mit einer speziellen zweistufigen Quecksilber-Dampfstrahlpumpe für die Zwischenkammer erlaubt, mit Düsen von je 0,2 mm Durchmesser bei einem Meßkammerdruck von $p_{0}=180$ Torr zu messen bzw. bei $p_{0}<50$ Torr eine rechteckige Öffnung von $10 \times 0,1 \mathrm{~mm}^{2}$ zwischen Meßkammer und Zwischen-

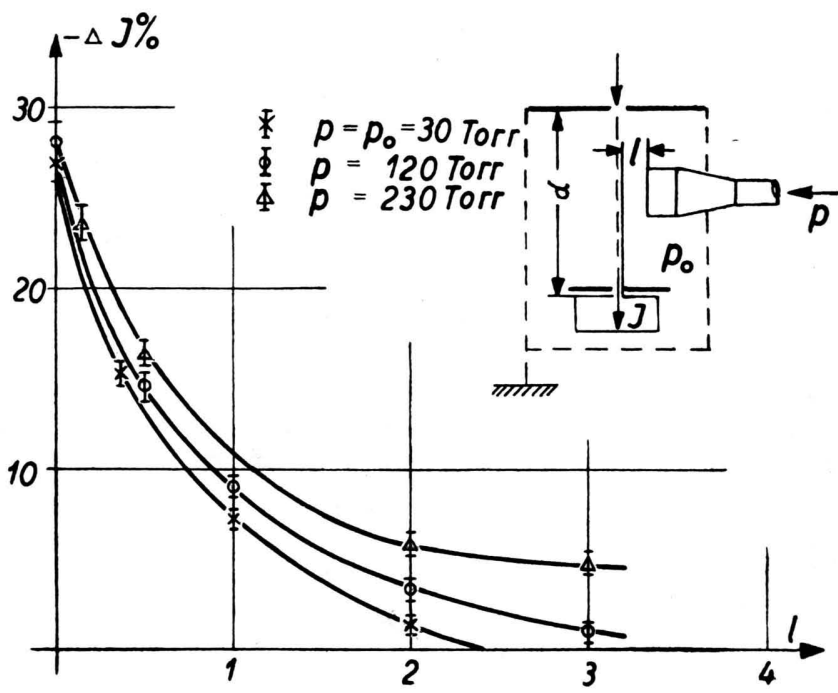

Abb. 3. Beispiel der Abtastung eines Luftstrahls mit der Elektronensonde.

kammer zu verwenden, in welcher der Sondenstrahl noch magnetisch abgelenkt werden kann, um das Meßfeld abzutasten. Mit einer zylindrischen Düse von $0,1 \mathrm{~mm}$ Durchmesser arbeitet das Rohr ohne Folie noch gegen einen Außendruck von 1,5 atm. Außerdem kann die Kathode bequem gegen eine Ionenquelle ausgewechselt werden.

Der Auffänger war ein kleiner abgeschirmter FaradayKäfig. Um zu verhindern, daß dieser, zusammen mit seiner Abschirmung oder der Wand des Meßraumes, eine Ionisationskammer bildet, mußte sein Potential durch eine Kompensationsschaltung auf $\pm 0,01 \mathrm{~V}$ genau auf gleichem Potential wie die Umgebung gehalten werden. Mitgemessene Ionenströme blieben dann unterhalb der Meßgenauigkeit. Ein konstanter Bruchteil von Sekundär-Elektronen kann dabei verlorengehen. Die Kompensation erfolgte z. Tl. mit Hilfe eines Fadenelektrometers von Hand, für rasch verlaufende Vorgänge wurde ein Kompensationsverstärker entwickelt.

Als erstes Beispiel für ein inhomogenes Dichtefeld sei die Vermessung eines Luftstrahls wiedergegeben, der einmal mit einem Druck $p=120$ Torr, einmal 
mit $p=230$ Torr in die Meßkammer einströmte, in der ein Druck $p_{0}=30$ Torr herrschte (Abb. 3); er trat aus einem Spalt von $10 \times 0,1 \mathrm{~mm}^{2}$ aus. Für verschiedene Entfernungen $l$ der Luftstrahldüse von der Sonde wurde die Intensität $J$ gemessen, der Luftstrahl also längs seiner Achse abgetastet. Auch ohne Luftstrahl tritt für kleine Entfernungen eine Abschattung der Elektronen ein, da die Düse in den Trübungsquerschnitt des Strahls eindringt. Sie wurde gesondert bestimmt. Abb. 3 zeigt $\Delta J \%$ als Funktion von $l$ für die drei Fälle, bezogen auf die Sondenintensität $\boldsymbol{J}_{0}$ am Auffänger ohne Düse und Luftstrahl. Für die verwendete Strahlenergie war $\alpha d=1$, somit nach $(7 \mathrm{~b}) \overline{\Delta \varrho} \% \approx-\Delta J \%$. Die strömende Gasmenge betrug bei $p=120$ Torr $Q_{1}=$ 13 Torr $\cdot l / \mathrm{sec}$, bei $p=230$ Torr $Q_{2}=30$ Torr $\cdot l /$ sec. Damit kommt die Strömungsgeschwindigkeit der Luft dicht vor der Düse schon im ersten Fall in die Größenordnung der Schallgeschwindigkeit; trotzdem ist der materielle "Strahl“ nach etwa $4 \mathrm{~mm}$ Weg wegdiffundiert. Über die Impulsfortpflanzung ist hiermit jedoch nichts ausgesagt. $\mathrm{Da}$ für $l=0$ nur ein kleiner Effekt zu finden ist, hängt mit dem Auflösungsvermögen der Sonde unter diesen Bedingungen zusammen.

Als weiteres Beispiel sind in Abb. 4 Messungen an einer Gebläseflamme wiedergegeben: Der Sondenstrahl, bestehend aus Elektronen von $50 \mathrm{kV}$ Strahlspannung und einer Intensität $J_{0} \approx 10^{-10} \mathrm{~A}$ trat ohne Folienfenster in die freie Atmosphäre aus und traf nach einer Meßstrecke $d=1,6 \mathrm{~cm}$ den Auffänger.

Die Flamme brannte in der Mitte zwischen des Austrittsdüse und dem Auffänger unter $45^{\circ}$ schräg von unten nach oben. Sie wurde in verschiedenen $\mathrm{Ab}$ ständen $l$ - von der Brennermündung gemessen von der Sonde durchsetzt. Aus der gemessenen Intensität $J_{l}$ ergibt sich die integrale Dichteänderung $\overline{\Delta \varrho}$ längs der Meßstrecke $d$, bezogen auf die Dichte $\varrho_{0}$ der Luft bei $20^{\circ} \mathrm{C}$ und die Sonden-Intensität $J_{0}$ am Auffänger ohne Flamme.

$$
\frac{\bar{J} \underline{Q}}{\varrho_{0}}=\frac{1}{{ }_{\varrho_{O_{0}}} d} \ln \frac{J_{l}}{J_{0}} .
$$

Diese Dichteänderung ist auf die heiße Zone in der Meßstrecke zu beziehen. Die Breite $s^{\prime}$ dieser - hier homogen angenommenen - heißen Zone ist aus den Abmessungen der Flamme ermittelt $\left[s^{\prime}(l)\right.$ in Abb. 4 , unterer Teil]. Sie könnte mit einer Sonde höheren geometrischen Auflösungsvermögens direkt gemessen werden.

Man erhält für die Dichte $\varrho l^{\prime}$ dieser Zone

$$
\frac{\Delta \varrho_{l}^{\prime}}{\varrho_{0}}=\frac{d}{s^{\prime}} \frac{\overline{\Delta \varrho}_{l}}{\varrho_{0}} .
$$

Das.nicht brennende Leuchtgas-Luft-Gemisch ergab keinen Dichteeffekt: Die relativ geringen Dichte-

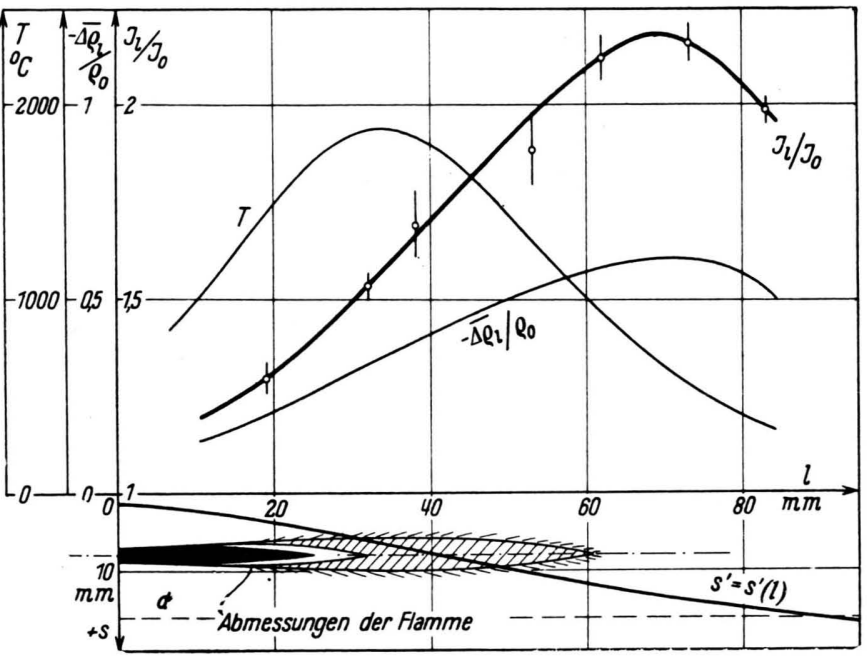

Abb. 4. Dichtemessung in einer Gebläseflamme mittels Elektronensonde.

änderungen liegen hier - im Gegensatz zu den Messungen von Abb. 3 - infolge der geringeren Strahlintensität $J_{0}$ unter der Ablesegenauigkeit.

Die gemessenen Dichteänderungen in der brennenden Flamme sind durch die jeweilige Flammentemperatur bedingt. Unter der Annahme konstanten Druckes ergibt sich qualitativ mit Gl. 8 der in Abb. 4 gezeichnete Temperaturverlauf $T$, der in diesem Falle natürlich auch direkt gemessen werden könnte, und bekannt ist.

Wir haben uns auf eine mehr qualitative Darstellung beschränkt. Über Einzelheiten und weitere Messungen, auch in Zusammenhang mit den eingangs erwähnten Problemen und mit Ionensonden, wird ander-weitig berichtet werden. 